\title{
Correction to: PD-1 axis expression in musculoskeletal tumors and antitumor effect of nivolumab in osteosarcoma model of humanized mouse
}

Bingxin Zheng ${ }^{1,2+}$, Tingting Ren ${ }^{1,2+}$, Yi Huang ${ }^{1,2}$, Kunkun Sun ${ }^{1,3}$, Shidong Wang ${ }^{1,2}$, Xing Bao ${ }^{1,2}$, Kuisheng Liu Li, $^{1,2}$ and Wei Guo ${ }^{1,2^{*}}$

\section{Erratum}

The original article [1] contained an error in Table 1 whereby the 'Positive' column in the 'PD-L1' Tumor type group of columns was mistakenly included at the beginning of the 'PD-L2' Tumor type group of columns.

This error has now been corrected. Furthermore, this error was not the fault of the authors, and was instead mistakenly carried forward by the production team that handled this article.

\begin{abstract}
Author details
${ }^{1}$ Musculoskeletal Tumor Center, Peking University People's Hospital, No. 11 Xizhimen South Street, Beijing 100044, People's Republic of China. ${ }^{2}$ Beijing Key Laboratory of Musculoskeletal Tumor, Beijing, People's Republic of China. ${ }^{3}$ Department of Pathology, Peking University People's Hospital, Beijing, People's Republic of China.
\end{abstract}

Received: 23 February 2018 Accepted: 23 February 2018 Published online: 12 March 2018

\section{Reference}

1. Zheng B. PD-1 axis expression in musculoskeletal tumors and antitumor effect of nivolumab in osteosarcoma model of humanized mouse. J Hematol Oncol. 2018;11:16

\footnotetext{
* Correspondence: bonetumor@pku.edu.cn

${ }^{\dagger}$ Equal contributors

${ }^{1}$ Musculoskeletal Tumor Center, Peking University People's Hospital, No. 11 Xizhimen South Street, Beijing 100044, People's Republic of China

${ }^{2}$ Beijing Key Laboratory of Musculoskeletal Tumor, Beijing, People's Republic of China

Full list of author information is available at the end of the article
} 
Table 1 Expression of PD-L1, PD-L2, and PD-1 in musculoskeletal tumors

\begin{tabular}{|c|c|c|c|c|c|c|c|c|c|c|c|c|c|c|c|c|}
\hline \multirow[t]{2}{*}{ Tumor } & \multirow[t]{2}{*}{$N$} & \multicolumn{5}{|c|}{$\begin{array}{l}\text { PD-L1 } \\
N(\%)\end{array}$} & \multicolumn{5}{|c|}{$\begin{array}{l}\text { PD-L2 } \\
N(\%)\end{array}$} & \multicolumn{5}{|c|}{$\begin{array}{l}\text { PD-1 } \\
N(\%)\end{array}$} \\
\hline & & 0 & $1+$ & $2+$ & $3+$ & Positive & $\overline{0}$ & $1+$ & $2+$ & $3+$ & Positive & $\overline{0}$ & $1+$ & $2+$ & $3+$ & Positive \\
\hline Musculoskeletal tumor & 234 & 179 & 35 & 10 & 10 & $55(23.5 \%)$ & 168 & 49 & 8 & 9 & $66(28.2 \%)$ & 185 & 37 & 5 & 7 & 49 (20.9\%) \\
\hline Giant cell tumor & 14 & 4 & 1 & 2 & 7 & $10(71.4 \%)$ & 7 & 1 & 1 & 5 & $7(50.0 \%)$ & 6 & 1 & 2 & 5 & $8(57.1 \%)$ \\
\hline Osteosarcoma & 62 & 40 & 17 & 3 & 2 & $22(35.5 \%)$ & 36 & 21 & 3 & 2 & $26(41.9 \%)$ & 45 & 15 & 1 & 1 & 17 (27.4\%) \\
\hline Synovial sarcoma & 127 & 107 & 16 & 3 & 1 & $20(15.7 \%)$ & 101 & 22 & 2 & 2 & $26(20.5 \%)$ & 103 & 21 & 2 & 1 & 24 (18.9\%) \\
\hline Chondrosarcoma & 31 & 28 & 1 & 2 & 0 & $3(9.7 \%)$ & 24 & 5 & 2 & 0 & $7(22.6 \%)$ & 31 & 0 & 0 & 0 & 0 \\
\hline Conventional chondrosarcoma & 27 & 27 & 0 & 0 & 0 & 0 & 21 & 4 & 2 & 0 & $6(22.2 \%)$ & 27 & 0 & 0 & 0 & 0 \\
\hline Dedifferentiated chondrosarcoma & 4 & 1 & 1 & 2 & 0 & $3(75.0 \%)$ & 3 & 1 & 0 & 0 & $1(25.0 \%)$ & 4 & 0 & 0 & 0 & 0 \\
\hline
\end{tabular}

